\title{
Novel genome characteristics contribute to the invasiveness of Phragmites australis
} (common reed)

Dong-Ha Oh ${ }^{1 凶}$, Kurt P. Kowalski ${ }^{2}$, Quynh N. Quach ${ }^{3}$, Chathura Wijesinghege ${ }^{1}$, Philippa Tanford $^{3,4}$, Maheshi Dassanayake ${ }^{1 凶}$, and Keith Clay ${ }^{3 \varpi}$

${ }^{1}$ Department of Biological Sciences, Louisiana State University, Baton Rouge, LA, USA. ${ }^{2}$ U.S. Geological Survey, Great Lakes Science Center, Ann Arbor, MI, USA. ${ }^{3}$ Department of Ecology \& Evolutionary Biology, Tulane University, New Orleans, LA, USA. ${ }^{4}$ Department of Biology, Washington University in St. Louis, St. Louis, MO, USA.

Correspondences: ohdongha@gmail.com, kkowalski@usgs.gov, maheshid@1su.edu, and clay@tulane.edu.

Keywords: Phragmites australis, Arundinoideae, whole genome duplication, reference genome, invasive, fungal inoculation, gene expression

\footnotetext{
Abstract

The rapid invasion of the non-native Phragmites australis (Poaceae, subfamily Arundinoideae) is a major threat to native ecosystems in North America. We describe a $1.14 \mathrm{Gbp}$ reference genome for P. australis and compare invasive (ssp. australis) and native (ssp. americanus) genotypes collected across the Laurentian Great Lakes to deduce genomic bases driving its invasive success. We report novel genomic features including a lineage-specific whole genome duplication, followed by gene loss and preferential retention of genes associated with transcription factors and regulatory functions in the remaining duplicates. The comparative transcriptomic analyses revealed that genes associated with biotic stress and defense responses were expressed at a higher basal level in invasive genotypes, but the native genotypes showed a stronger induction of defense responses following fungal inoculation. The reference genome and transcriptomes, combined with previous ecological and environmental data, support the development of novel, genomics-assisted management approaches for invasive Phragmites.
} 


\section{Introduction}

Invasion of native ecosystems by non-native species is a worldwide problem damaging to ecosystems and economies. Invasive plants can negatively affect agricultural production ${ }^{1,2}$ and displace native species through multiple mechanisms including enemy release ${ }^{3,4}$, allelopathy ${ }^{5,6}$, and novel traits ${ }^{7,8}$. Thus, management and control of invasive plants can be a priority for conservation and agriculture.

Phragmites australis (Cav.) Trin. ex Steud.(Common Reed, Poaceae) is globally distributed (Fig. 1a) and provides multiple ecosystem services in its native range ${ }^{9-11}$. A native subspecies ( $P$. australis ssp. americanus) has been present in North American wetlands for thousands of years ${ }^{12}$. However, the non-native, invasive subspecies (P. australis ssp. australis) ${ }^{13,14}$ was introduced to North America from Europe around 1900 and has been aggressively disrupting and displacing native plant communities ${ }^{13,15}$ and altering wildlife habitat and ecosystem properties ${ }^{16,17}$. The invasive subspecies occurs throughout the contiguous United States (U.S.) and the entire Great Lakes basin ${ }^{13,18,19}$ (Fig. 1b). It is one of the most problematic invasive plant species in wetland habitats in eastern North America, with hundreds of millions of dollars per year invested in control efforts ${ }^{20,21}$. It is more robust than the native subspecies (Fig. 1d,e), with larger inflorescences, leaves, and height (Fig. 1f,g), but both subspecies reproduce by seed and clonally via rhizomes (Fig. 1h). A variety of mechanisms promoting P. australis invasions have been proposed, but effective control strategies are lacking ${ }^{22}$. The Great Lakes Restoration Initiative identified invasive species (including $P$. australis) as one of its five most urgent issues ${ }^{23}$. Invasive $P$. australis has also been recognized as the leading plant model for studying genetic mechanisms underlying plant invasions ${ }^{21,24}$. P. australis therefore provides an excellent system to test genetic adaptations and control measures in plant invasions as both native and invasive populations coexist over a large geographic range (Fig. 1a,b).

$P$. australis exhibits a range of ploidy levels from $2 \mathrm{x}-12 \mathrm{x}$, with tetraploids reported as dominant in Europe and North America and octoploids dominant in Asia ${ }^{25,26}$. P. australis genotypes, ploidy levels, and genome size have been assessed for traits that may favor invasiveness such as photosynthetic rate and nitrogen use efficiency, rhizome sizes, shoot emergence rates, and herbivory resistance ${ }^{27-29}$. However, $P$. australis lacks a reference genome that can serve as a foundational resource to investigate genomic traits underlying plant invasions and to identify genetic targets for biocontrol. Further, Phragmites belongs to the grass subfamily 
Arundinoideae, which has been poorly explored at the genomic level compared to other grass subfamilies, even though some species are ecologically dominant or invasive on a global scale ${ }^{30}$.

We report here the first reference genome for the invasive Phragmites australis ssp. australis, as well as comparative genomic and transcriptomic analyses that include invasive and native genotypes coexisting in the Great Lakes region of North America. Our results provide a key genomic resource for grasses and the subfamily Arundinoideae, novel insights into evolution in an underexplored grass clade, biological pathways correlated with invasiveness, and a genomic foundation for development of new management approaches. 


\section{Results}

\section{Assembly and annotation of the common reed Phragmites australis}

We sequenced the genome of an invasive genotype of Phragmites australis ssp. australis collected in the U.S. Fish and Wildlife Service, Ottawa National Wildlife Refuge, Ohio (Fig. 1c, star-marked). We obtained $\sim 42.19 \mathrm{Gbp}$ of high confidence sequence data from leaf genomic DNA, representing 37-fold genome coverage, using PacBio SMRT sequencing technology. Assembled using Canu ${ }^{31}$ (version 1.4; see Methods), the reference genome provides 1.14 Gbp of 13,411 gap-free contigs with more than half of the assembled genome captured in 1,370 contigs (N50) larger than $194.6 \mathrm{kbp}$ (L50). The largest contig is $3.22 \mathrm{Mbp}$ (Table 1). Illumina short reads mapped to the primary assembly confirmed the genotype used as the reference being diploid based on single nucleotide polymorphisms that represented a non-reference allele frequency distribution with a peak at 0.5, as expected with a diploid genome (Supplementary Fig. 1). In total, $56.19 \%$ of the genome consist of repetitive sequences, with sequences derived from long terminal repeat (LTR) retrotransposons constituting $36.42 \%$ of the genome (Supplementary Table 1). Based on the repeat-masked genome sequence, we annotated 64,857 protein-coding gene models with a total length of $72.35 \mathrm{Mbp}$, which accounted for $6.35 \%$ of the genome (Table 1). The genomic location of these gene models and their best orthologs in rice and Arabidopsis thaliana are provided in Supplementary Dataset 1. BUSCO (Benchmarking Universal SingleCopy Orthologs) analysis ${ }^{32}$ found $93.3 \%$ of single-copy gene models expected for land plants in the Phragmites reference genome (Supplementary Table 2).

We constructed a species tree using 6,404 gene models representing 782 gene families from P. australis and 13 other published grass genomes (PLAZA monocot database v. 4.5) ${ }^{33}$, with pineapple (Ananas comosus, Bromeliaceae) as the outgroup. P. australis, representing the first genome from the Arundinoideae subfamily, was placed sister to subfamily Chloridoideae, consistent with the PACMAD clade species tree (Fig. 2) ${ }^{34,35}$.

\section{Signatures of a recent whole genome duplication in the $P$. australis genome}

We found signatures of a previously unreported whole genome duplication in the $P$. australis genome that occurred after its divergence from the subfamily Panicoideae (Fig. 3). We compared the $P$. australis genome with five representative monocot reference genomes, including pineapple (A. comosus) and four grass species without a genome duplication more 
recent than the $\rho$ duplication ${ }^{36}$ (Fig. 2, marked with asterisks). The $P$. australis genome presented $36.7 \%$ BUSCO genes as duplicated, while only 1-5\% BUSCOs were duplicated in the five comparator genomes (Fig. 3a and Supplementary Table 2).

A genome-wide alignment between Setaria italica and P. australis found that $50.2 \%$ of $S$. italica gene models are represented twice in the $P$. australis genome as co-linear orthologs in synteny blocks (Fig. 3a, and Supplementary Figure 2a). Similar patterns were observed in comparisons between $P$. australis and the other four monocot genomes (Supplementary Figure 2a).

A genome-wide self-alignment using SynMap identified 14,005 gene loci, comprising $21.6 \%$ of all $P$. australis protein-coding genes, that were organized into 1,501 paralogous synteny blocks consisted of at least five co-linear paralog pairs. Synteny blocks were widespread across the $P$. australis genome and found in $66.8 \%$ of all contigs with ten or more protein-coding gene loci (Supplementary Fig. 2b and c), further supportive of whole genome duplication (WGD) events.

To assess the timing of genome duplications that resulted in the observed paralogous synteny blocks, we used neutral evolutionary substitutions calculated for 4-fold degenerate (4D) sites in codons that allows positioning of timing of duplicated events within a clade. We plotted the distribution of synonymous substitution rate $(K s)$ at 4D sites for P. australis co-linear paralog pairs and for co-linear ortholog pairs between P. australis and comparator species. (Fig. 3c). The peak $K s$ for P. australis co-linear paralog pairs (Fig. 3c, dark gray) was smaller than those observed for any pairwise comparisons between species, while larger than the value found between P. australis ssp. australis and native P. australis ssp. americanus (Fig. 3c, bottom row). We also found a small proportion of synteny blocks within the P. australis genome that likely represent the $\rho$ duplication at the root of the Poaceae (Supplementary Fig. 3). Our analysis suggests that the majority of co-linear paralogs in synteny blocks are derived from a single WGD event that occurred after the divergence of Phragmites from the Panicoideae, represented here by S. italica and S. bicolor, but before the divergence between the subspecies australis and americanus (Fig. 3d).

\section{Biased retention of transcription factors and signaling-related genes following the lineage- specific genome duplication in $P$. australis}


While the synteny blocks are widespread across the $P$. australis genome, the relatively short length of each synteny block (Supplementary Fig. 2c) and the fact that only $21.6 \%$ of duplicated genes were retained in synteny blocks indicates a substantial fractionation following genome duplication, likely due to the selective retention of duplicates that enhance plant fitness and loss of redundant or detrimental duplicates. We therefore compared the functions of duplicated $P$. australis genes relative to those genes with conserved copy numbers based on ortholog groups including other grass species (Fig. 4).

For all ortholog groups (OGs) detected among P. australis and the five comparator monocot species (Methods and Supplementary Dataset 2), we calculated the ortholog copy number ratio $\left(R_{C N}\right)$ for each species by dividing the copy number in the species with the average of the other five species (Fig. 4a and Supplementary Table 3). All species except Phragmites showed a peak at $R_{C N} \approx 1$. By contrast, the $R_{C N}$ distribution in $P$. australis showed two peaks, one with $R_{C N}$ at 1 (Fig. 4a, "Conserved") and a second peak at $R_{C N} \approx 2$ (Fig. 4a, "Duplicated"), indicating that a substantial number of $P$. australis OGs have their copy numbers doubled compared to other species (See Methods for details). We searched for enriched gene functions in the Duplicated group (11,002 P. australis genes in 4,113 OGs) and in the Conserved group $(6,981$ P. australis genes in 5,600 OGs). Fig. 4b, c present the five largest functional clusters, detected using GOMCL ${ }^{37}$, considering GO terms significantly enriched exclusively in either Conserved or Duplicated groups. The largest clusters included $72 \%$ and $64 \%$ of all Phragmites genes represented by 123 and 146 GO terms enriched in the Duplicated and the Conserved groups, respectively (Supplementary Dataset 3). Functions enriched exclusively in the Duplicated group were largely related to regulation of gene expression (Fig. 4b, c). For example, "transcription regulator activity" is the most enriched functional cluster among duplicated genes (Fig. 4b, c, cluster D1), representing 10.6\% of all genes in the Duplicated group with a GO annotation, compared to only $3.5 \%$ and $8.1 \%$ in the Conserved group and all genes (used as the background for the enrichment analysis), respectively (Supplementary Dataset 3). By contrast, the Conserved group was enriched in functions associated with primary metabolic processes and transport (C1-5 in Fig 4b, c and Supplementary Dataset 3). Selective gene retention after WGD events are often linked to adaptive traits or traits leading to lineage diversification. The biased retention of transcription factors and regulatory processes in P. australis spp. australis following 
its most recent WGD event is indicative of greater genomic plasticity conducive to invasive lifestyles ${ }^{38}$.

\section{Divergence in basal transcriptome profiles between invasive and native $\boldsymbol{P}$. australis} subspecies

To gain further insight into genetic factors promoting invasiveness, we compared transcriptomes of invasive and native Phragmites subspecies collected from the Great Lakes region (Fig. 1c, 5a). We generated eight replicates of RNA-seq samples from leaf and rhizome tissues, respectively (see Methods), followed by independent de novo transcriptome assembly of the six genotypes (Supplementary Table 4). The maximum likelihood tree based on concatenated alignments of 3,464 homolog groups separated the six genotypes into invasive and native subspecies as expected, and the three invasive genotypes were placed together with the invasive genotype used as the reference genome (Fig. 5b).

We aligned RNA-seq reads to the reference genome to explore the differences in basal transcriptome profiles between invasive and native genotypes and estimate the relative abundance of reference genes, as detailed in Methods (Supplementary Dataset 4). The three native genotypes showed overall $8.86 \%$ fewer reads aligned to the reference genome, compared to the three invasive genotypes, which showed an $88.92 \pm 4.21 \%$ alignment rate on average (Supplementary Table 5). When normalized to the total number of aligned reads, the distribution of estimated expression values did not show a noticeable bias towards either invasive or native genotypes (Supplementary Fig. 4). The leaf- and rhizome-derived transcriptomes were distinct from each other in a principal component analysis (PCA) (on PC1), while both showed additional separation between native and invasive genotypes (on PC2) (Fig 5c). In agreement with the PCA, the number of differentially expressed genes (DEGs) between invasive and native genotypes was much greater than DEGs detected between any other genotype comparisons (Fig. $5 \mathrm{~d})$. On average, $11.5 \pm 1.0 \%$ of the 64,857 reference gene models showed higher basal expression in the invasive genotypes compared to the native genotypes (Fig. $5 \mathrm{~d}$, upper right sections of the diagonal plots). Similarly, $12.1 \pm 2.1 \%$ of gene models were more highly expressed in the native genotypes than in the invasive genotypes (Fig. 5d, lower left sections).

In the leaf samples, "response to stimulus" and "response to stress" were the largest representative GO terms showing significant enrichment among DEGs with higher basal 
expression in the invasive genotypes compared to the native genotypes in all cross-genotype comparisons (Supplementary Dataset 5). Interestingly, among child GO terms of these two GO terms, only those categorized as "response to biotic stimulus" and "defense response" showed significant enrichment in the invasive genotypes, but not "response to abiotic stress" (Supplementary Fig. 5). On average, 9.2\% of all DEGs were annotated with "defense response" and showed higher basal expression in the invasive genotypes compared to $6.4 \%$ and $6.2 \%$ in the native genotypes and the background, respectively (Fig. 5e, upper panel). This bias towards higher a basal expression of genes associated with biotic stress and defense in the invasive genotypes was less clear in the rhizome tissue (Fig. 5e, lower panel). By contrast with the functional enrichment in defense responses observed for the invasive genotypes, the native genotypes were biased towards genes associated with "transmembrane transport" and its child GO terms, including "ammonium transport”, are represented by DEGs with higher basal expression in all cross-genotype comparisons (Fig. 5f and Supplementary Dataset 5). This transcriptomic signal was more prominent in leaf samples compared to rhizomes as similarly observed for the defense response.

\section{Divergence in invasive and native $P$. australis transcriptomic responses to biotic stress induced by fungal endophyte inoculation}

We inoculated target genotypes with the fungal endophyte commonly isolated from $P$. australis, Alternaria alternata ${ }^{39}$ (see Methods), to further investigate the transcriptomic signal established at basal expression contrasting the invasive genotypes from native genotypes biased towards biotic stress responses (Fig. 6, 1c, 5a). We compared 24 RNA-seq profiles generated for leaf and rhizome tissue harvested at pre-inoculation (basal expression) and post-inoculation (response to biotic stress) to deduce DEGs and their representative enriched functions in individual genotypes (see Methods, Supplementary Dataset 4). In general, more DEGs were significantly induced (Fig. 6a) than repressed (Fig. 6b) in response to the endophyte inoculation. The two invasive genotypes IOH1 and IMI1 showed more attenuated responses to endophyte inoculation compared to the three native genotypes. However, the invasive genotype IMI3 showed a response similar in magnitude to the native genotype NMI2 (Fig. 6a,b and Supplementary Dataset 6). Further, IMI3 and the three native genotypes shared a substantial number of endophyte-induced DEGs (Fig. 6a red boxes). 
We searched for enriched functional associations between endophyte-induced and repressed genes in the invasive and native genotypes (Fig. 6c and Supplementary Dataset 6). In the native genotypes and IMI3 genotype, endophyte-induced genes were enriched in "ATP binding" and "defense response", while endophyte-repressed genes were enriched in processes associated with photosynthesis (Fig. 6c). A closer inspection into these induced genes annotated under GO Molecular Function, "ATP-binding”, and GO Biological Process, "defense response”, revealed that many encode membrane receptor kinases known for roles in defense signaling (Supplementary Dataset 6).

The transcriptomic responses following endophyte inoculation may be reflective of local adaptations defined by each site selected for our study, compounding the broad distinctions we are able to deduce between native and invasive genotypes. For example, genotypes IMI3 and NMI2, while showing the highest number of induced DEGs annotated under defense response, also showed the highest number of repressed DEGs associated with photosynthesis compared to the rest of the genotypes, suggesting that the strong defense response concurrently downregulated photosynthesis in these two genotypes (Fig. 6c-e). This potential trade-off related to photosynthesis seems to be less prominent in NOH1 where both the number and enrichment of photosynthesis-related endophyte-repressed DEGs were among the smallest compared to other genotypes. Despite genotypic variation in response to the endophyte inoculation, we found that in the native genotypes there was an overall stronger response in induced genes associated with biotic stress and defense, while these functions had higher basal expression in the invasive genotypes. 


\section{Discussion}

The genome of common reed, Phragmites australis reported here provides the first reference genome for the ecologically dominant and invasive model grass, spp. australis ${ }^{13,14}$, and the first reference genome for the grass subfamily Arundinoideae (Fig. 1, 2, Table 1). Our genomic analyses, including non-reference allele frequency distributions and comparative genomics with other grasses, indicate that the reference genome was derived from a functionally diploid plant ${ }^{40}$, despite $P$. australis being generally considered a tetraploid in North America ${ }^{25}$. However, we found a previously unreported whole genome duplication (WGD) event, independent of three ancient WGD events known in the grasses ${ }^{36}$, suggestive of P. australis being a paleo-tetraploid. The paleo-WGD event detected in the $P$. australis genome predates the divergence between spp. australis and americanus, but postdates the divergence from the Panicoideae (Fig. 3 and 4). As expected with substantial gene fractionation following WGD events, the $P$. australis genome lost up to $48 \%$ of its duplicated genes (Supplementary Fig. 2a), but retained over 14,005 duplicated genes. The retained duplicated genes were strongly enriched with transcription factors and other genes associated with regulatory processes. Genes encoding transcription factors were preferentially retained over other gene groups following paleo WGD events in pan-global species, such as Arabidopsis thaliana ${ }^{41-43}$, and is thought to be a hallmark feature of WGD events underlying rapid diversification and global distribution of angiosperms 44,45

Adaptive innovations initiated at the genomic level further diversified at the transcriptome level can lead to distinct ecological fates. Our comparative analysis using native and invasive genotypes from the Great Lakes region indicates that gene expression associated with defense against biotic stress is primed in the invasive genotypes compared to native genotypes, and when a biotic stress response was induced using a fungal endophyte, the native genotypes were consistently more responsive (Fig. 5, 6). P. australis is known for its intraspecific ploidy polymorphism ${ }^{25,46}$ coincident with its extreme success, being a globally recognized invasive species. Whether ploidy plays a deterministic role in facilitating invasiveness in $P$. australis remains debatable when categorical higher ploidy levels exclusively assigned to invasive genotypes are absent. However, as proposed by previous studies, it may predispose a species for invasive lifestyles adapted to a broad range of habitats potentially defined by both biotic and abiotic stresses ${ }^{47}$. Therefore, exploring $P$. australis pangenomes in 
future studies should provide further insight into how ploidy polymorphism could add selective advantages to invasive genotypes of $P$. australis over their closely related non-invasive genotypes. Finally, our results provide a foundation for the development of novel species- and subspecies-specific genetic approaches for control of invasive Phragmites ${ }^{48}$. There is widespread interest in controlling invasive Phragmites in many parts of its invasive range. Our genomic data help to identify particular genes that might be targeted in genetic control approaches such as RNAi, but our results also provide some caution in particular approaches if targeted genes are duplicated. While RNAi approaches have been widely explored for controlling invasive insect pests and plant pathogens ${ }^{49,50}$, to our knowledge, no RNAi-based treatments have been developed to control problematic plants given the dearth of genomic data from invasive plant species. Future attempts at genetic control for invasive Phragmites should take into account that genetic variation exists in invasive Phragmites genotypes and that native genotypes can co-occur in the same habitats. 


\section{Methods}

Plant materials. For genome sequencing, tillers and associated rhizome tissues were collected from a single $P$. australis clump of chloroplast haplotype $\mathrm{M}^{13,14}$ at the Ottawa National Wildlife Refuge near Toledo, Ohio (Fig. 1c, marked with "G") and propagated in a walk-in growth chamber as detailed in Supplementary Methods. For transcriptome analyses, we collected three additional invasive and three native genotypes from four sites around the Great Lakes in Michigan and Ohio, U.S. (Fig. 1c and Fig. 5a). Native genotypes were readily distinguished from invasive genotypes by their smaller stature and thinner tillers with distinctive reddish coloration at the nodes (Fig. 1e, f, g). Both native and invasive genotypes were confirmed by chloroplast haplotype sequences as previously described ${ }^{13,51}$. Phragmites plants were grown in the growth chamber and subjected to endophyte inoculation treatments before RNA isolation for RNA-seq analyses, as detailed in Supplementary Methods.

Genome sequencing, assembly, and annotation. Genomic DNA was isolated from leaf tissue using a $2 \%$ CTAB extraction protocol ${ }^{52}$, converted to SMRTbell ${ }^{\mathrm{TM}}$ libraries (Pacific Bioscience, Menlo Park, CA) with 20-Kb target insert size, and sequenced for continuous long reads (CLR) using a PacBio RSII single-molecule real-time sequencing platform. After post-processing (SMRT Analysis v2.3, Pacific Bioscience), total 4.79 million reads (mean length $8.80 \mathrm{Kbp}$, N50 13.11 Kbp, and total $42.19 \mathrm{Gbp}$ ) were assembled into contigs using Canu assembler (v. 1.4) ${ }^{31}$, with the target genome size set to $1 \mathrm{Gbp}$, generating the reference genome assembly. In addition, the same genomic DNA samples were converted to TruSeq DNA paired-ends libraries (Illumina, San Diego, CA) for whole-genome shotgun sequencing, analyzed for 300 cycles on a NextSeq platform (Illumina), and used to estimate the genome ploidy levels (Supplementary Methods and Supplementary Fig. 1).

For genome annotation, we first used RepeatModeler v. 1.0.8 and RepeatMasker v. 4.0.9 (http://www.repeatmasker.org/) to detect transposable elements and repetitive sequences in the assembled $P$. australis contigs. De novo-detected repetitive sequences were combined with known monocot repeat sequences in RepBase (v. 23.07; http://www.girinst.org/) to mask repeats in $P$. australis contigs. Protein-coding gene models on the repeat-masked genome were predicted using the MAKER (v. 2.31.10) ${ }^{53}$ with P. australis BUSCO-trained parameters for ab initio gene model prediction ${ }^{32}$ and hints from $P$. australis transcriptomes de novo assembled using Trinity 
(v. 2.1.1) ${ }^{54}$ as well as from homologs from Sorghum (Phytozome ID:454) and Brachypodium (Phytozome ID:314). In addition, we performed a reference-guided transcriptome assembly using StringTie (v. 2.0.1) ${ }^{55}$ to report putative isoform models. The gene model encoding the longest open reading frame (ORF) was selected as the representative for each of the 64,857 protein-coding gene loci.

Comparative and functional analyses. We used BUSCO (v. 3$)^{32}$ to assess the completeness of the representative $P$. australis protein-coding gene models in comparison with grass genomes available in Monocots PLAZA (v. 4.5) ${ }^{33}$ (Supplementary Table 2). Maximum-likelihood species trees were estimated by OrthoFinder (v. 2.2.7) ${ }^{56}$ and RAxML (v. 8.2) ${ }^{57}$ using concatenated alignment of 782 sequences. We selected five monocot genomes for in-depth comparative analyses, based on their availability of recently updated gene models with $>90 \%$ BUSCO scores and lack of genome duplication more recent than the $\rho$ event. MCscan (as implemented in JCVI v. 1.1.7) ${ }^{58}$ was used to compare syntenic depths between $P$. australis and other monocot genomes. SynMap ${ }^{59}$ and CLfinder pipeline ${ }^{60}$ detected co-linear paralog and ortholog pairs within the $P$. australis genome and between $P$. australis and monocot genomes, respectively. We estimated synonymous substitution rates at 4-fold degenerate sites using codeml ${ }^{61}$ as described previously ${ }^{60}$. We detected ortholog groups among representative gene models of $P$. australis and other monocot genomes using OrthoFinder (v. 2.2.7) ${ }^{56}$ and MMseqs $2{ }^{62}$, and subsequently identified P. australis ortholog groups that are "Conserved" and "Duplicated" in ortholog copy numbers compared to other monocot species, as detailed in Supplementary Methods.

Gene ontology (GO) annotation was transferred to the Phragmites representative gene models based on sequence similarities to plant proteins with GO annotations as of January $1^{\text {st }}$, 2020 in the GO consortium (http://geneontology.org/). In short, Phragmites protein sequences were compared with reference sequences with a GO annotation using MMseqs2 with the maximum sensitivity (-s 7.5). If the protein alignment covers minimum $30 \%$ of both the query and subject sequences, the GO annotation was transferred to the Phragmites protein. We used BiNGO ${ }^{63}$ to detect GO terms enriched in Phragmites gene models. GO terms were further clustered and summarized using GOMCL ${ }^{37}$. 
Endophyte treatment, transcriptome assembly, and RNA-seq analyses. Invasive and native genotypes of $P$. australis were propagated from rhizome cuttings, grown in a growth chamber for 60 days, and subjected to Alternaria alternata (accession KT923239) ${ }^{39}$ fungal endophyte inoculation and RNA-Seq, as detailed in Supplementary Methods. RNA-seq reads were filtered of adapter sequences using FASTP ${ }^{64}$. Filtered reads were aligned to the reference genome using HISAT2 (v. 2.2) and expression of each gene model was estimated with StringTie (v. 2.0.1) with default parameters ${ }^{65}$. Differentially Expressed Genes (DEGs) were identified based on a minimum 2-fold difference in expression levels, with adjusted p-values $<0.05$ (estimated by DESeq2 ${ }^{66}$ ), between pairs of genotypes or between endophyte-inoculated and control samples within each genotype. In addition, putative protein-coding transcript sequences were obtained from filtered RNA-Seq reads by the Trinity (v. 2.1.1) and TransDecoder (v. 5.5.0) pipeline ${ }^{54}$ (Supplementary Table 4) and used for estimating the phylogenetic relationship of P. australis subspecies and genotypes using the Agalma pipeline (v. 2) ${ }^{67}$ (Fig. $3 \mathrm{~d}$ and 5b).

\section{Data availability}

All raw and assembled sequence data are deposited to NCBI under BioProject PRJNA705976. In addition, the reference genome sequence, gene models, and representative RNA-Seq tracks are available in the CoGe database with the Genome ID 59768, for browsing and other CoGeembedded comparative analyses ${ }^{68}$. 


\section{Acknowledgements}

The authors would like to thank The U.S. Fish and Wildlife Service and Michigan Department of Environment, Great Lakes and Energy for access to study sites and the Great Lakes Restoration Initiative for financial support. We thank Doug Rusch and Ram Podicheti and the staff of the Indiana University Center for Genomics and Bioinformatics for their help in initial genome and transcriptome analyses, Matt Filapek for lab assistance, and the Indiana University Greenhouse staff for their assistance with growing and propagating Phragmites. We thank Dr. Wesley Bickford for data collection and analysis support and Drs. Ping Gong, Kathleen Ferris, and Simon Barak for their thoughtful comments. This research was supported by the United States Geological Survey Cooperative Agreement G18AC00373 to KC. MD and DHO acknowledge the support of National Science Foundation awards MCB-1616827, NSF-IOS-EDGE-1923589, and the Next-Generation BioGreen21 Program of Republic of Korea (PJ01317301). CW was supported by an Economic Development Assistantship award from Louisiana State University. The authors also acknowledge the LSU High Performance Computing services for providing computational resources needed for data analyses.

\section{Author contributions}

K.C., M.D., and K.K. conceived and designed the experiments and obtained the funding. P.T., K.C., and K.K. conducted data collections and related experiments. D-H.O., C.W, and MD designed the bioinformatics work flows and performed computational analyses. D-H.O and Q.Q. generated figures. All authors contributed to drafting and finalizing the manuscript.

\section{Competing Interests}

The authors declare no competing interests. Any use of trade, product, or firm names is for descriptive purposes only and does not imply endorsement by the U.S. Government. 


\section{References}

1. Pimentel, D., Zuniga, R. \& Morrison, D. Update on the environmental and economic costs associated with alien-invasive species in the United States. Ecol. Econ. 52, 273-288 (2005).

2. Dogra, K. S., Sood, S. K., Dobhal, P. K. \& Sharma, S. Alien plant invasion and their impact on indigenous species diversity at global scale: A review. J. Ecol. Nat. Environ. 2, 175-186 (2010).

3. Keane, R. M. \& Crawley, M. J. Exotic plant invasions and the enemy release hypothesis. Trends Ecol. Evol. 17, 164-170 (2002).

4. Mitchell, C. E. \& Power, A. G. Release of invasive plants from fungal and viral pathogens. Nature 421, 625-627 (2003).

5. Chengxu, W., Mingxing, Z., Xuhui, C. \& Bo, Q. Review on allelopathy of exotic invasive Plants. Procedia Eng. 18, 240-246 (2011).

6. Kalisz, S., Kivlin, S. N. \& Bialic-Murphy, L. Allelopathy is pervasive in invasive plants. Biol. Invasions (2020) doi:10.1007/s10530-020-02383-6.

7. Kolar, C. S. \& Lodge, D. M. Progress in invasion biology: predicting invaders. Trends Ecol. Evol. 16, 199-204 (2001).

8. Divíšek, J. et al. Similarity of introduced plant species to native ones facilitates naturalization, but differences enhance invasion success. Nat. Commun. 9, 4631 (2018).

9. Rooth, J. E. \& Stevenson, J. C. Sediment deposition patterns in Phragmites australis communities: Implications for coastal areas threatened by rising sea-level. Wetlands Ecol. Manage. 8, 173-183 (2000).

10. Whitaker, K. et al. Vegetation persistence and carbon storage: Implications for environmental water management for Phragmites australis. Water Resour. Res. 51, 52845300 (2015).

11. Kiviat, E. Ecosystem services of Phragmites in North America with emphasis on habitat functions. AoB Plants 5, (2013).

12. Saltonstall, K., Peterson, P. M. \& Soreng, R. J. Recognition of Phragmites australis subsp. americanus (Poaceae: Arundinoideae) in North America: Evidence from morphological and genetic analyses. SIDA Contrib. Bot. 21, 683-692 (2004).

13. Saltonstall, K. Cryptic invasion by a non-native genotype of the common reed, Phragmites australis, into North America. Proc. Natl. Acad. Sci. U. S. A. 99, 2445-2449 (2002). 
14. Martin, L. J. \& Blossey, B. The Runaway Weed: Costs and failures of Phragmites australis management in the USA. Estuaries Coast. 36, 626-632 (2013).

15. Mozdzer, T. J., Brisson, J. \& Hazelton, E. L. G. Physiological ecology and functional traits of North American native and Eurasian introduced Phragmites australis lineages. AoB Plants 5, (2013).

16. Rogalski, M. A. \& Skelly, D. K. Positive effects of nonnative invasive Phragmites australis on larval bullfrogs. PLoS One 7, e44420 (2012).

17. Perez, A., Mazerolle, M. J. \& Brisson, J. Effects of exotic common reed (Phragmites australis) on wood frog (Lithobates sylvaticus) tadpole development and food availability. J. Freshw. Ecol. 28, 165-177 (2013).

18. Tulbure, M. G. \& Johnston, C. A. Environmental conditions promoting non-native Phragmites australis expansion in Great Lakes coastal wetlands. Wetlands 30, 577-587 (2010).

19. Bourgeau-Chavez, L. L. et al. Mapping invasive Phragmites australis in the coastal Great Lakes with ALOS PALSAR satellite imagery for decision support. J. Great Lakes Res. 39, 65-77 (2013).

20. Kowalski, K. P. et al. Advancing the science of microbial symbiosis to support invasive species management: a case study on Phragmites in the Great Lakes. Front. Microbiol. 6, 95 (2015).

21. Meyerson, L. A., Cronin, J. T. \& Pyšek, P. Phragmites australis as a model organism for studying plant invasions. Biol. Invasions 18, 2421-2431 (2016).

22. Hazelton, E. L. G., Mozdzer, T. J., Burdick, D. M., Kettenring, K. M. \& Whigham, D. F. Phragmites australis management in the United States: 40 years of methods and outcomes. AoB Plants 6, (2014).

23. Great Lake Restoration Initiative. Great Lake Restoration Initiative (GLRI) Action Plans. (2019).

24. Cesarino, I. et al. Plant science's next top models. Ann. Bot. 126, 1-23 (2020).

25. Clevering, O. A. \& Lissner, J. Taxonomy, chromosome numbers, clonal diversity and population dynamics of Phragmites australis. Aquat. Bot. 64, 185-208 (1999).

26. Saltonstall, K. Microsatellite variation within and among North American lineages of Phragmites australis. Mol. Ecol. 12, 1689-1702 (2003). 
27. Pyšek, P. et al. Competition among native and invasive Phragmites australis populations: An experimental test of the effects of invasion status, genome size, and ploidy level. Ecol. Evol. 10, 1106-1118 (2020).

28. Park, M. G. \& Blossey, B. Importance of plant traits and herbivory for invasiveness of Phragmites australis (Poaceae). Am. J. Bot. 95, 1557-1568 (2008).

29. Guo, W.-Y., Lambertini, C., Nguyen, L. X., Li, X.-Z. \& Brix, H. Preadaptation and postintroduction evolution facilitate the invasion of Phragmites australis in North America. Ecol. Evol. 4, 4567-4577 (2014).

30. Grass Phylogeny Working Group et al. Phylogeny and subfamilial classification of the grasses (Poaceae). Ann. Mo. Bot. Gard. 88, 373-457 (2001).

31. Koren, S. et al. Canu: scalable and accurate long-read assembly via adaptive k-mer weighting and repeat separation. Genome Res. 27, 722-736 (2017).

32. Waterhouse, R. M. et al. BUSCO Applications from quality assessments to gene prediction and phylogenomics. Mol. Biol. Evol. 35, 543-548 (2018).

33. Van Bel, M. et al. PLAZA 4.0: an integrative resource for functional, evolutionary and comparative plant genomics. Nucleic Acids Res. 46, D1190-D1196 (2018).

34. Burke, S. V. et al. Evolutionary relationships in Panicoid grasses based on plastome phylogenomics (Panicoideae; Poaceae). BMC Plant Biol. 16, 140 (2016).

35. Hardion, L. et al. Cleaning up the grasses dustbin: systematics of the Arundinoideae subfamily (Poaceae). Plant Syst. Evol. 303, 1331-1339 (2017).

36. McKain, M. R. et al. A phylogenomic assessment of ancient polyploidy and genome evolution across the Poales. Genome Biol. Evol. 8, 1150-1164 (2016).

37. Wang, G., Oh, D.-H. \& Dassanayake, M. GOMCL: a toolkit to cluster, evaluate, and extract non-redundant associations of Gene Ontology-based functions. BMC Bioinformatics 21, 139 (2020).

38. Clark, J. W. \& Donoghue, P. C. J. Whole-Genome Duplication and Plant Macroevolution. Trends Plant Sci. 23, 933-945 (2018).

39. Clay, K., Shearin, Z. R. C., Bourke, K. A., Bickford, W. A. \& Kowalski, K. P. Diversity of fungal endophytes in non-native Phragmites australis in the Great Lakes. Biol. Invasions 18, 2703-2716 (2016).

40. Li, Z. et al. Patterns and Processes of Diploidization in Land Plants. Annu. Rev. Plant Biol. 
(2021) doi:10.1146/annurev-arplant-050718-100344.

41. Blanc, G. \& Wolfe, K. H. Functional divergence of duplicated genes formed by polyploidy during Arabidopsis evolution. Plant Cell 16, 1679-1691 (2004).

42. Freeling, M., Rapaka, L., Lyons, E., Pedersen, B. \& Thomas, B. C. G-boxes, bigfoot genes, and environmental response: characterization of intragenomic conserved noncoding sequences in Arabidopsis. Plant Cell 19, 1441-1457 (2007).

43. De Bodt, S., Maere, S. \& Van de Peer, Y. Genome duplication and the origin of angiosperms. Trends Ecol. Evol. 20, 591-597 (2005).

44. Birchler, J. A. Genomic Balance Plays Out in Evolution. Plant Cell 31, 1186-1187 (2019).

45. Cheng, F. et al. Gene retention, fractionation and subgenome differences in polyploid plants. Nat Plants 4, 258-268 (2018).

46. Lambertini, C. et al. A phylogeographic study of the cosmopolitan genus Phragmites (Poaceae) based on AFLPs. Plant Syst. Evol. 258, 161-182 (2006).

47. te Beest, M. et al. The more the better? The role of polyploidy in facilitating plant invasions. Ann. Bot. 109, 19-45 (2012).

48. Harvey-Samuel, T., Ant, T. \& Alphey, L. Towards the genetic control of invasive species. Biol. Invasions 19, 1683-1703 (2017).

49. Cagliari, D. et al. Management of Pest Insects and Plant Diseases by Non-Transformative RNAi. Front. Plant Sci. 10, 1319 (2019).

50. Mamta, B. \& Rajam, M. V. RNAi technology: a new platform for crop pest control. Physiol. Mol. Biol. Plants 23, 487-501 (2017).

51. Saltonstall, K. The naming of Phragmites haplotypes. Biol. Invasions 18, 2433-2441 (2016).

52. Doyle, J. J. \& Dickson, E. E. Preservation of plant samples for DNA restriction endonuclease analysis. Taxon 36, 715-722 (1987).

53. Campbell, M. S., Holt, C., Moore, B. \& Yandell, M. Genome Annotation and Curation Using MAKER and MAKER-P. Curr. Protoc. Bioinformatics 48, 4.11.1-39 (2014).

54. Haas, B. J. et al. De novo transcript sequence reconstruction from RNA-seq using the Trinity platform for reference generation and analysis. Nat. Protoc. 8, 1494-1512 (2013).

55. Pertea, M. et al. StringTie enables improved reconstruction of a transcriptome from RNAseq reads. Nat. Biotechnol. 33, 290-295 (2015). 
56. Emms, D. M. \& Kelly, S. OrthoFinder: phylogenetic orthology inference for comparative genomics. Genome Biol. 20, 238 (2019).

57. Stamatakis, A. RAxML-VI-HPC: maximum likelihood-based phylogenetic analyses with thousands of taxa and mixed models. Bioinformatics 22, 2688-2690 (2006).

58. Tang, H. et al. Synteny and collinearity in plant genomes. Science 320, 486-488 (2008).

59. Lyons, E. \& Freeling, M. How to usefully compare homologous plant genes and chromosomes as DNA sequences. Plant J. 53, 661-673 (2008).

60. Oh, D.-H. \& Dassanayake, M. Landscape of gene transposition-duplication within the Brassicaceae family. DNA Res. 26, 21-36 (2019).

61. Yang, Z. PAML: a program package for phylogenetic analysis by maximum likelihood. Comput. Appl. Biosci. 13, 555-556 (1997).

62. Steinegger, M. \& Söding, J. MMseqs2 enables sensitive protein sequence searching for the analysis of massive data sets. Nat. Biotechnol. 35, 1026-1028 (2017).

63. Maere, S., Heymans, K. \& Kuiper, M. BiNGO: a Cytoscape plugin to assess overrepresentation of gene ontology categories in biological networks. Bioinformatics 21, 3448-3449 (2005).

64. Chen, S., Zhou, Y., Chen, Y. \& Gu, J. fastp: an ultra-fast all-in-one FASTQ preprocessor. Bioinformatics 34, i884-i890 (2018).

65. Pertea, M., Kim, D., Pertea, G. M., Leek, J. T. \& Salzberg, S. L. Transcript-level expression analysis of RNA-seq experiments with HISAT, StringTie and Ballgown. Nat. Protoc. 11, 1650-1667 (2016).

66. Love, M. I., Huber, W. \& Anders, S. Moderated estimation of fold change and dispersion for RNA-seq data with DESeq2. Genome Biol. 15, 550 (2014).

67. Dunn, C. W., Howison, M. \& Zapata, F. Agalma: an automated phylogenomics workflow. BMC Bioinformatics 14, 330 (2013).

68. Nelson, A. D. L., Haug-Baltzell, A. K., Davey, S., Gregory, B. D. \& Lyons, E. EPIC-CoGe: managing and analyzing genomic data. Bioinformatics 34, 2651-2653 (2018).

69. Barrero, R. A. et al. Shoot transcriptome of the giant reed, Arundo donax. Data Brief 3, 1-6 (2015). 
Figures and Tables (in the order of appearance in the text)

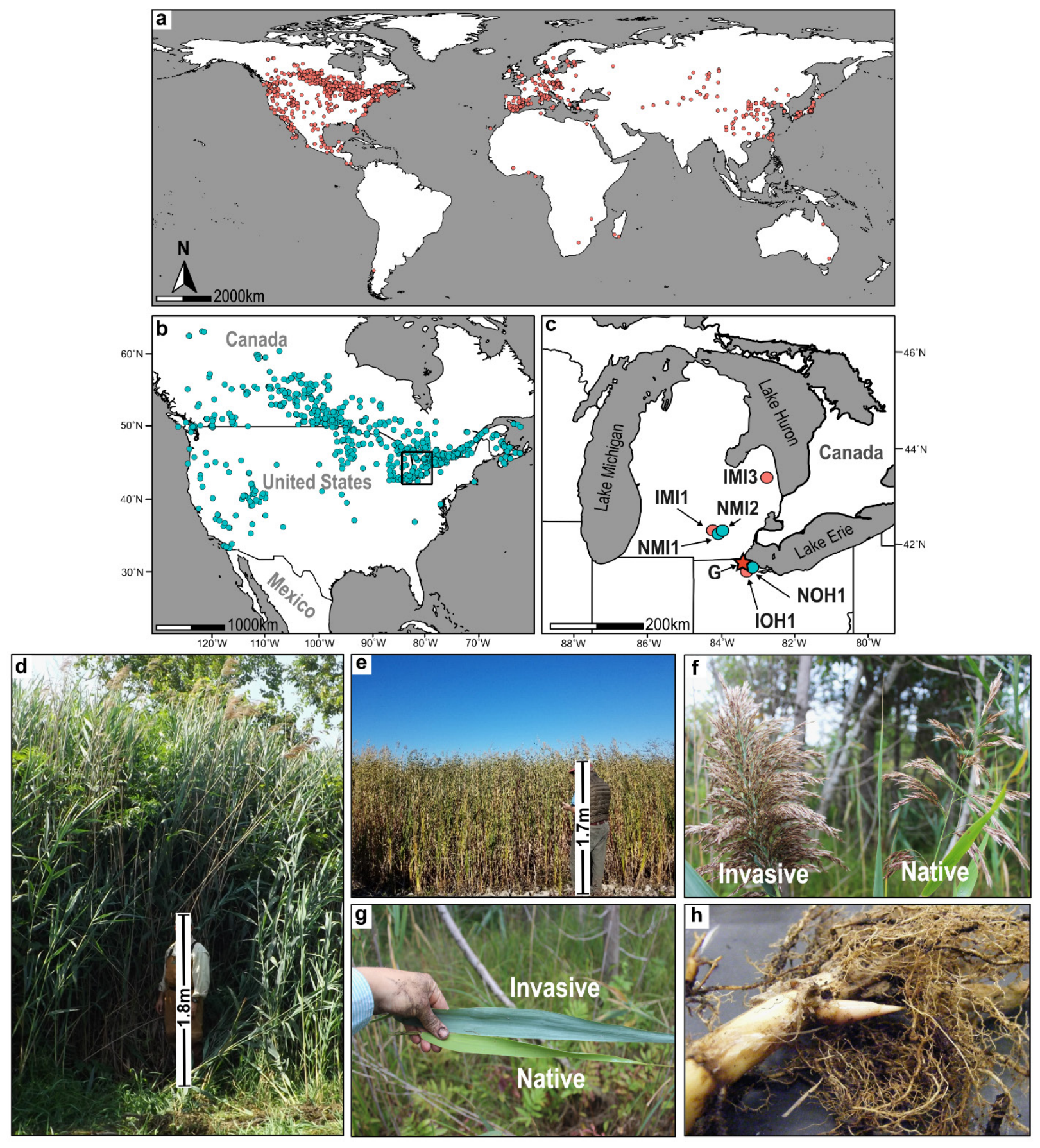

Fig. 1 | The invasive and native subspecies of common reed (Phragmites australis). a,

Reported global distribution of Phragmites australis ssp. australis ("Invasive") and b, Reported distribution of $P$. australis ssp. americanus ("Native") in the U.S.A. and Canada based on the Global Biodiversity Information Facility (https://www.gbif.org/, data access September 2020). c, A magnified view of the box in panel b showing sample site locations in Michigan (MI) and Ohio $(\mathrm{OH})$ in the U.S.A. Invasive (I) and Native (N) Phragmites plants were sampled from three 
Michigan sites (MI1, MI2, and MI3) and one Ohio site (OH1) in the Great Lakes region. Clonal fragments for the reference genome $(\mathrm{G})$ were collected near the OH1 site. See Fig. 5a for more details. d, Invasive P. australis ssp. australis stand growing in a western Lake Erie coastal wetland. e, Native P. australis ssp. americanus stand located in a western Lake Erie wetland. f, Seed heads from the invasive and native $P$. australis growing in Michigan. g, Invasive and native $P$. australis leaves collected in Michigan. $\mathbf{h}$, Rhizome and dense fibrous roots from an invasive $P$. australis plant growing in Michigan. 
Table 1 | The Phragmites australis draft genome

Assembly

Assembled genome size

1,139,927,050 bps

Largest contig size

$3,219,705$ bps

N50 contig length (L50)

194,574 bps

Total number of contigs

13,411

Number of contigs $>1 \mathrm{Mbps}$

67

Number of contigs $>$ N50

1,370

\section{Annotation}

Number of protein-coding gene loci

64,857

Total length of predicted ORFs

$72,347,598$ bps

Longest ORF

14,790 bps

Median length of ORFs

927 bps

Proportion of ORFs in the genome

$6.35 \%$

Proportion of repeats in the genome

$56.19 \%$

SINEs

$0.14 \%$

LINEs

$1.74 \%$

LTR elements

$36.42 \%$

DNA elements

$11.43 \%$

Unclassified repeats

$6.46 \%$ 


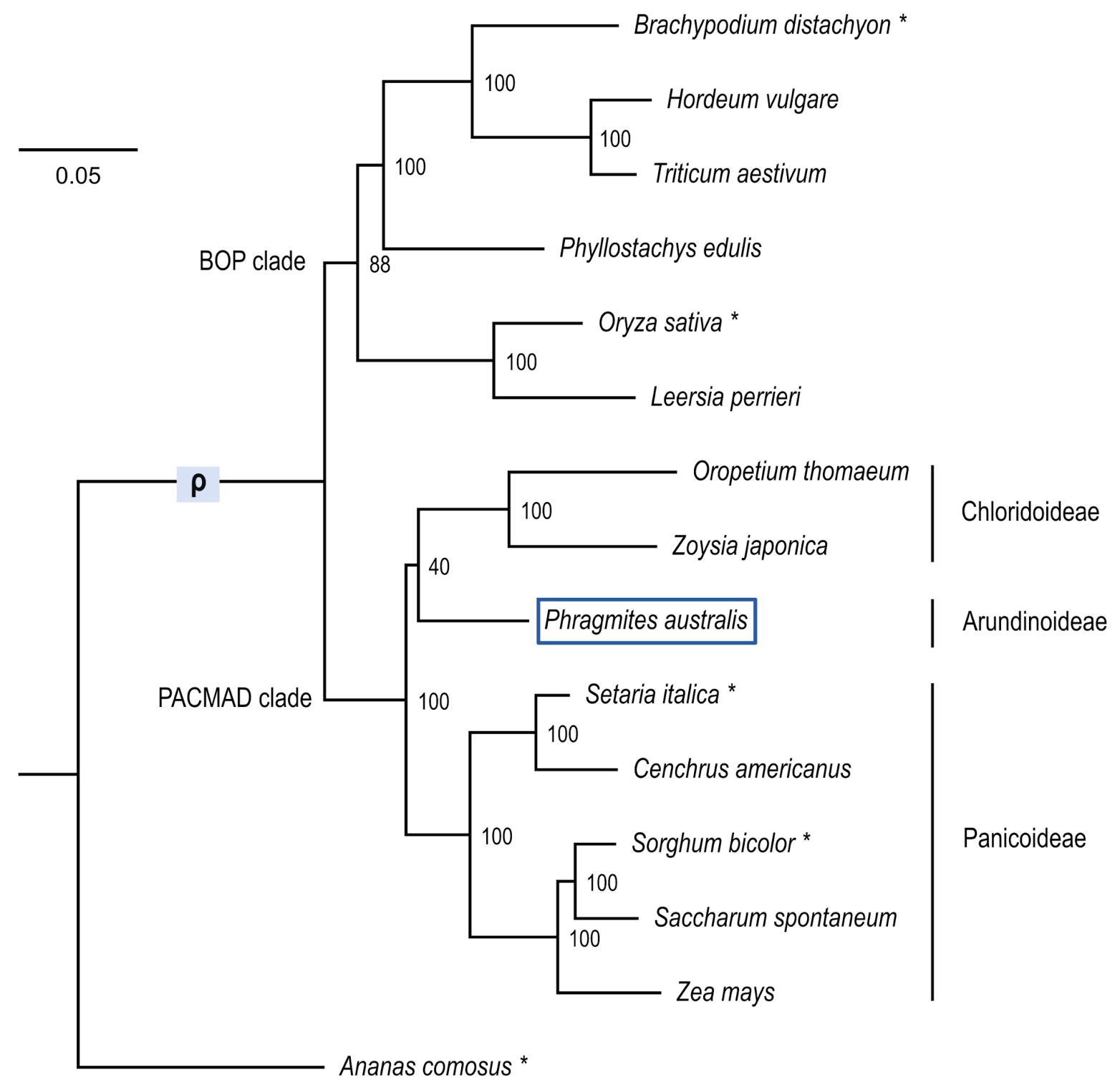

Fig. 2 | Phylogenetic position of Phragmites australis in the under-investigated

Arundinoideae subfamily, based on the draft genome. For the $P$. australis draft genome and 14 monocot genomes publicly available in Monocots PLAZA (v. 4.5) ${ }^{33}$, a maximum likelihood species tree was constructed based on 26,878 amino acid alignments from 782 ortholog groups, selected based on the criteria that at least seven species among the set had a single ortholog. All sites that included gaps in more than $20 \%$ of taxa were excluded. The number in each branch shows percent support from 1000 bootstrap replicates. The branch with the genome duplication 36 is marked, and species with asterisks were used for comparative analyses. 


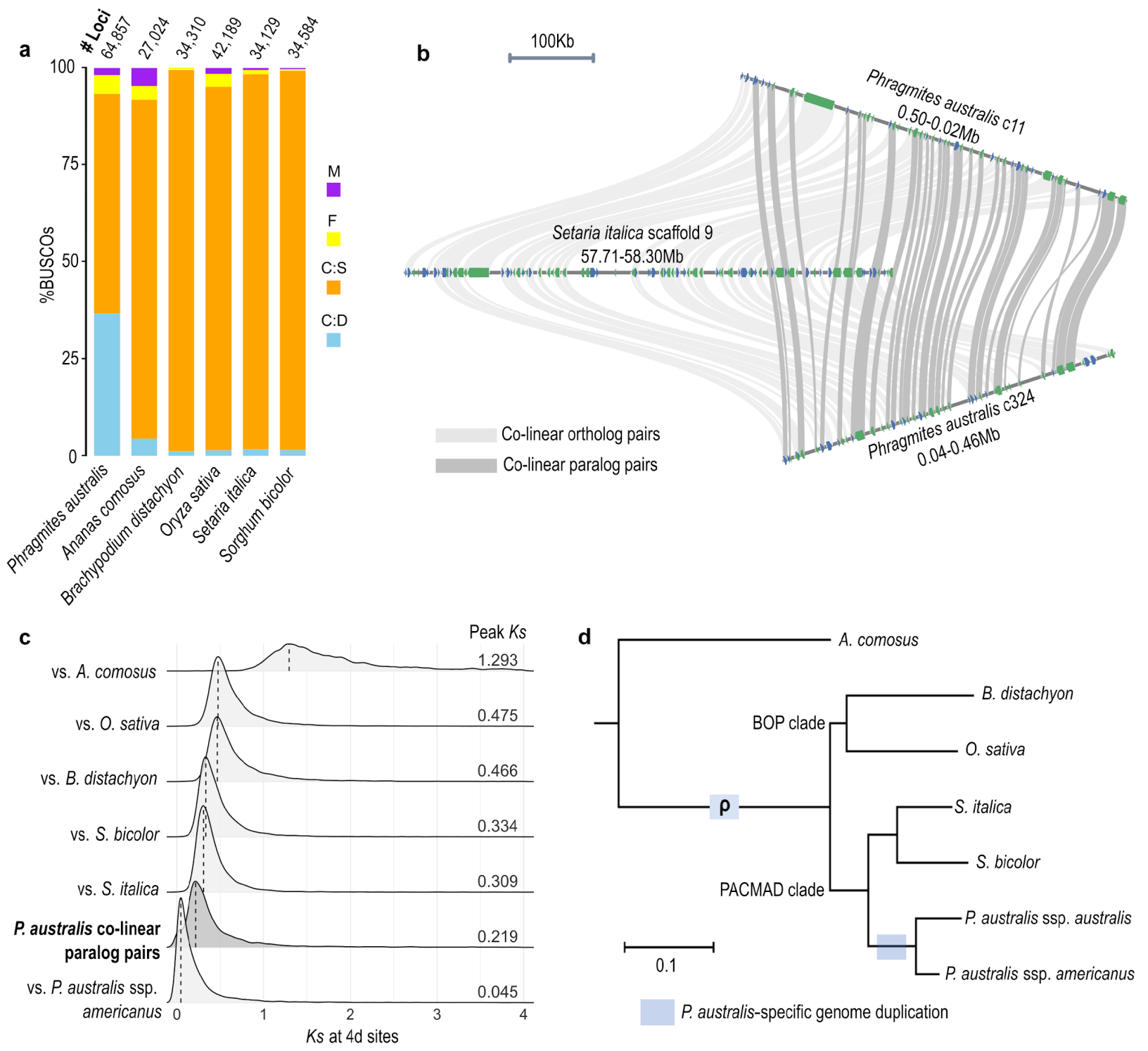

Fig. 3 | Signatures of Phragmites australis-specific whole genome duplication (WGD). a, Percentages of complete duplicated (C:D), complete single-copy $(C: S)$, fragmented $(F)$, and missing (M) orthologs among 1,375 Benchmarking Universal Single-Copy Orthologues (BUSCOs) and the number of protein-coding gene loci (\# Loci), in the genomes of $P$. australis and other monocot species. b, An example microsynteny between a 500-Kb Setaria italica genomic block and two duplicated $P$. australis genomic blocks. Ribbons connect co-linear ortholog (light gray) and paralog (dark gray) pairs identified by $\mathrm{MCscan}^{58}$ as described in Methods. c, Synonymous substitution rates $(K s)$ at 4-fold degenerate (4d) sites were estimated for co-linear ortholog pairs between $P$. australis and A. comosus (total 2,720 ortholog pairs), $B$. distachyon (10,559 pairs), O. sativa (11,176 pairs), S. italica (12,878 pairs), and S. bicolor (12,322 pairs), respectively, as well as 6,600 co-linear paralog pairs detected within the $P$. australis genome. For comparison with the native $P$. australis ssp. americanus, we used 11,445 reciprocal best homolog pairs between $P$. australis reference genome and a de novo 
bioRxiv preprint doi: https://doi.org/10.1101/2021.04.19.440155; this version posted April 20, 2021. The copyright holder for this preprint (which

was not certified by peer review) is the author/funder. This article is a US Government work. It is not subject to copyright under 17 USC 105 and is also made available for use under a CCO license.

transcriptome assembly from $P$. australis ssp. americanus. Probability distributions and peak values of $K s$ are shown. d, A maximum- likelihood species tree shows the branches associated with the $P$. australis-specific WGD, as well as the $\rho$ WGD event shared among grasses ${ }^{36}$. 
a

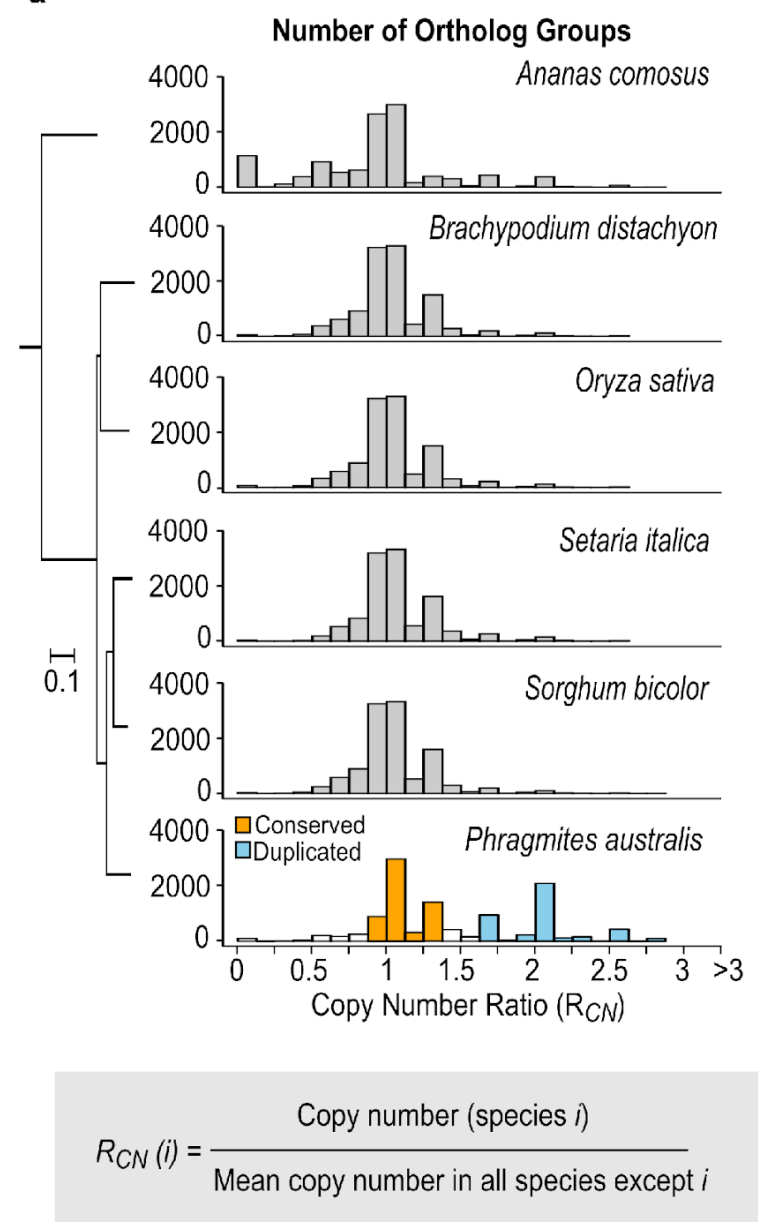

\section{b}
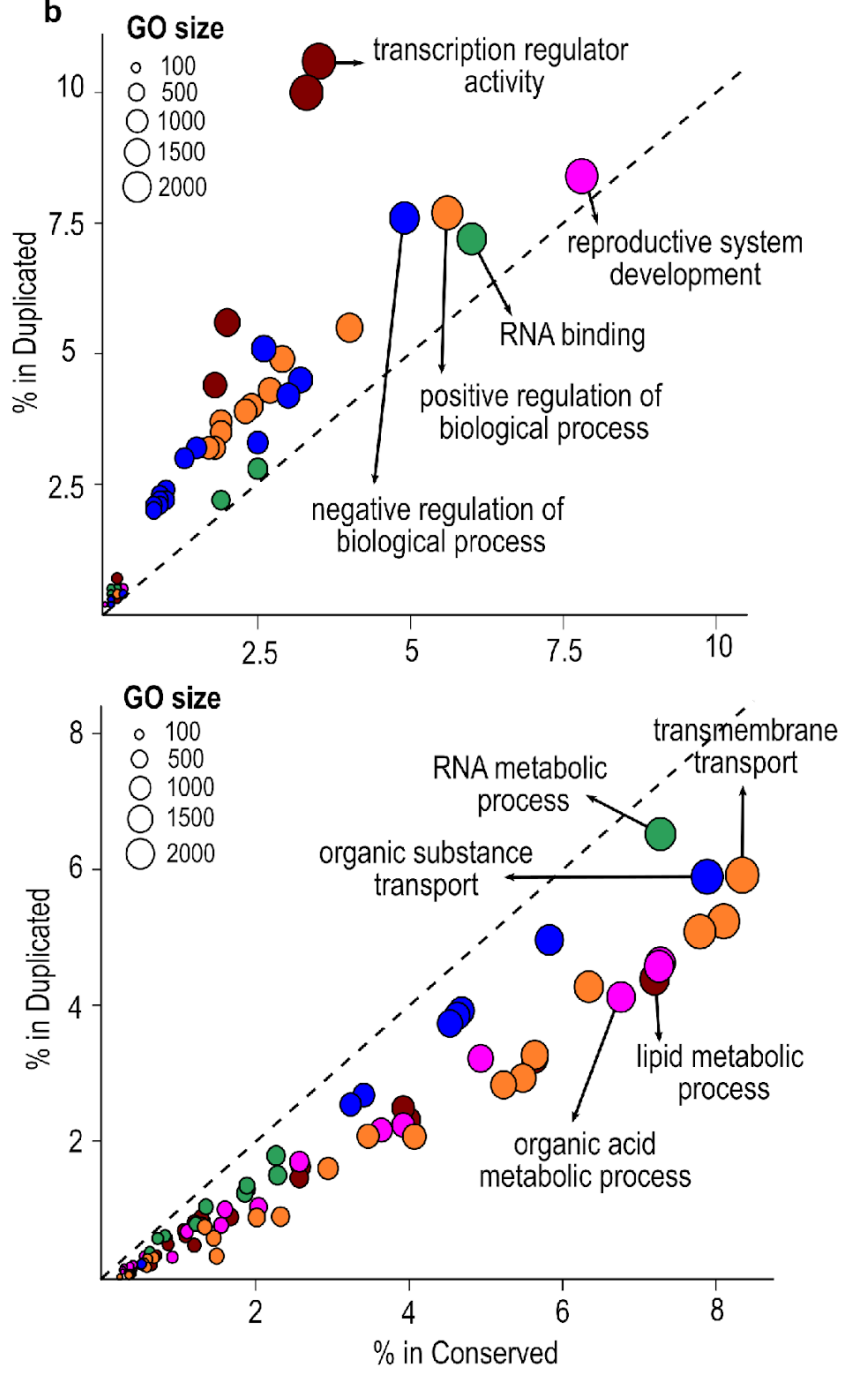

C

\begin{tabular}{|c|c|c|c|c|c|c|}
\hline \multirow[b]{2}{*}{ Cluster } & \multirow[b]{2}{*}{ 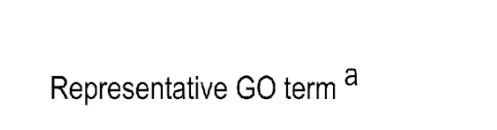 } & \multicolumn{2}{|c|}{ Duplicated } & \multicolumn{2}{|c|}{ Conserved } & \multirow{2}{*}{$\frac{\text { Background }}{\text { Genes (\%) }}$} \\
\hline & & Genes (\%) $b$ & $P^{C}$ & Genes $(\%)^{b}$ & $P^{C}$ & \\
\hline D1 & transcription regulator activity & $834(10.6)$ & $1.9 \mathrm{E}-16$ & $182(3.5)$ & - & $2963(8.1)$ \\
\hline $\mathrm{D} 2$ & reproductive system development & $665(8.4)$ & $1.9 \mathrm{E}-2$ & $408(7.8)$ & $6.6 \mathrm{E}-1$ & $2772(7.5)$ \\
\hline D3 & RNA binding & $567(7.2)$ & $7.8 \mathrm{E}-5$ & $309(6.0)$ & $7.3 \mathrm{E}-1$ & $2197(6.0)$ \\
\hline D4 & positive regulation of biological process & $616(7.7)$ & $6.5 \mathrm{E}-3$ & $293(5.6)$ & - & $2524(6.8)$ \\
\hline D5 & negative regulation of biological process & $608(7.6)$ & $1.9 \mathrm{E}-8$ & $259(4.9)$ & - & $2244(6.1)$ \\
\hline $\mathrm{C} 1$ & lipid metabolic process & $349(4.4)$ & - & $378(7.2)$ & $1.5 \mathrm{E}-6$ & $2017(5.5)$ \\
\hline $\mathrm{C} 2$ & organic acid metabolic process & $368(4.6)$ & - & $382(7.3)$ & $1.4 \mathrm{E}-6$ & $2040(5.5)$ \\
\hline $\mathrm{C} 3$ & RNA metabolic process & $519(6.5)$ & $5.4 \mathrm{E}-2$ & $382(7.3)$ & $6.4 \mathrm{E}-4$ & $2172(5.9)$ \\
\hline $\mathrm{C} 4$ & transmembrane transport & $471(5.9)$ & - & $438(8.3)$ & $3.3 \mathrm{E}-2$ & $2693(7.3)$ \\
\hline C5 & organic substance transport & $469(5.9)$ & - & $414(7.9)$ & $8.3 \mathrm{E}-3$ & $2467(6.7)$ \\
\hline
\end{tabular}


Fig. 4 | Functions enriched among $P$. australis genes that remained duplicated after the WGD event. a, Comparison of ortholog copy numbers between $P$. australis and five other monocot species. For each ortholog group identified by OrthoFinder as described in Methods, Copy Number Ratio $\left(R_{C N}\right)$ was calculated for each species by dividing the ortholog number in the species with the mean ortholog copy number in the other five species. Histograms show a shift towards increased $R_{C N}$ uniquely in P. australis. We identified "Conserved" (orange) and "Duplicated" (sky-blue) groups among $P$. australis genes whose copy numbers remain unchanged and uniquely increased, respectively, compared to other monocot species. b-c, GO terms enriched in either Duplicated or Conserved P. australis gene groups. The proportion of genes annotated with each GO term in Duplicated and Conserved groups was plotted as circles in (b). GO terms at least $80 \%$ overlapping with a bigger GO term are clustered and the largest five GO clusters enriched in either group were shown with the same color in (b) and (c).

${ }^{a}$ The largest GO term in each GO cluster; MF, Molecular Function; BP, Biological Process. ${ }^{b}$ Percentages are to the total number of genes with a $\mathrm{GO}$ annotation in each group. ${ }^{\mathrm{c}} \mathrm{P}$-values of enrichment compared to the Background, after Benjamini-Hochberg correction for multiple testing. Values less than 0.05 are in bold. 
bioRxiv preprint doi: https://doi.org/10.1101/2021.04.19.440155; this version posted April 20, 2021. The copyright holder for this preprint (which was not certified by peer review) is the author/funder. This article is a US Government work. It is not subject to copyright under 17 USC 105 and is also made available for use under a CCO license.

a

\begin{tabular}{|c|c|c|c|}
\hline ID & Genotype & Description & Coordinates \\
\hline IMI1 & Invasive & Railroad, Garvey Rd, Chelsea, MI, USA & $42.307211,-84.059662$ \\
\hline NMl1 & Native & Private, Garvey Rd, 50 meters SW from IMl1 site & $42.307135,-84.060249$ \\
\hline NMl2 & Native & Private, McKinley Rd, Chelsea, MI, USA & $42.337980,-84.00040$ \\
\hline IMI3 & Invasive & $\begin{array}{l}\text { Sandusky State Game Area, Michigan Dept. Natural Resources, } \\
\text { Sandusky, MI, USA }\end{array}$ & $43.394026,-82.790088$ \\
\hline $\mathrm{IOH} 1$ & Invasive & $\begin{array}{l}\text { Show Pool, U.S. Fish and Wildlife Service, Ottawa National Wildlife } \\
\text { Refuge, Oak Harbor, OH, USA }\end{array}$ & $41.614505,-83.195807$ \\
\hline $\mathrm{NOH} 1$ & Native & Show Pool, 110 meters NW from IOH1 site & $41.614755,-83.197046$ \\
\hline G & Invasive & Show Pool, 3 kilometers from $\mathrm{IOH} 1$ site & $41.631281,-83.228892$ \\
\hline
\end{tabular}

b

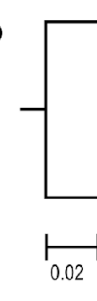

Sorghum bicolor

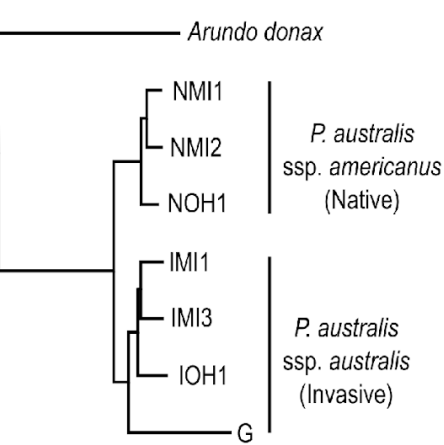

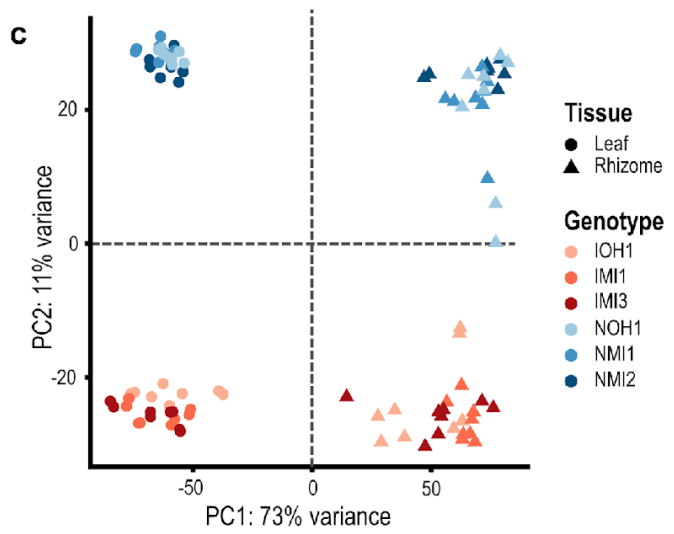

e

(Red > Blue in each cell)

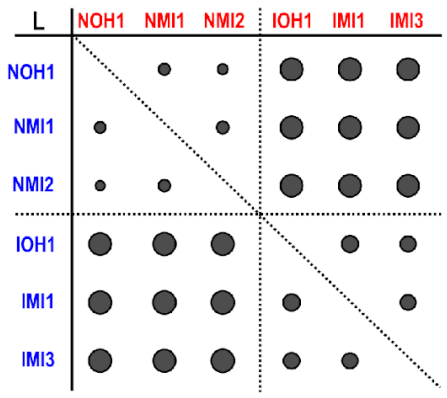

\begin{tabular}{l|lll:lll}
$\mathrm{R}$ & NOH1 & NMI1 & NMI2 & IOH1 & IMI1 & IMI3 \\
\hline
\end{tabular}

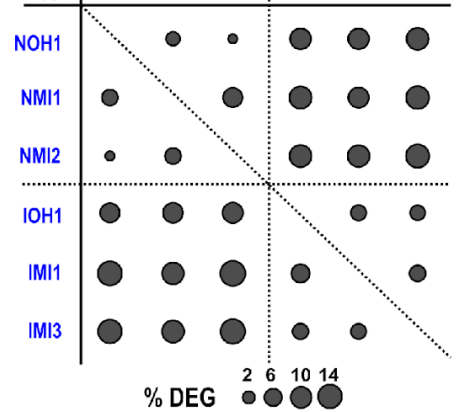

GO: defense response (6.2\% in background) in DEGs (Red > Blue in each cell)

f GO: transmembrane transport (6.5\% in background) in DEG
(Red $>$ Blue in each cell)

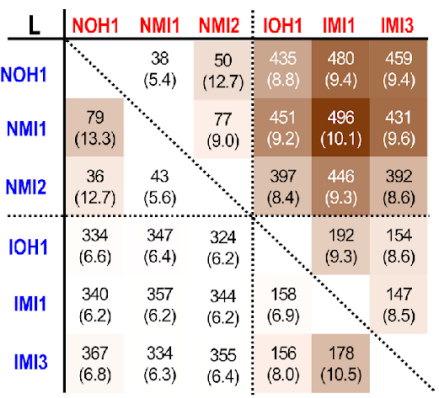

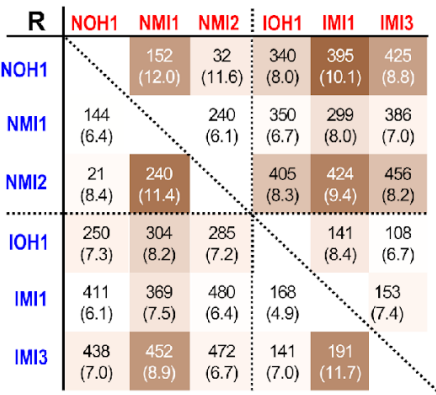

-Log 10 Padj Enrichment: 0 \begin{tabular}{llll|l|l|l}
1.3 & 5 & 10 & 15 & 20 & 25
\end{tabular}

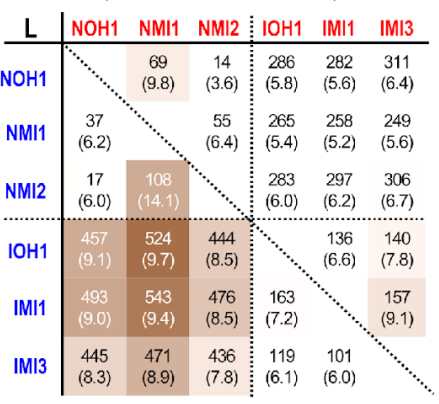

\begin{tabular}{l|lll:lll}
$\mathbf{R}$ & NOH1 & NMI1 NMI2 & IOH1 IMI1 & IMI3 \\
\hline
\end{tabular}

\begin{tabular}{|c|c|c|c|c|c|c|}
\hline $\mathrm{NOH} 1$ & & $\begin{array}{r}119 \\
(9.4)\end{array}$ & $\begin{array}{c}15 \\
(5.4)\end{array}$ & $\begin{array}{l}200 \\
(4.7)\end{array}$ & $\begin{array}{l}239 \\
(6.1)\end{array}$ & $\begin{array}{l}251 \\
(5.2)\end{array}$ \\
\hline NMI1 & $\begin{array}{c}104 \\
(4.6)\end{array}$ & & & $\begin{array}{l}242 \\
(4.6)\end{array}$ & $\begin{array}{r}199 \\
(5.3)\end{array}$ & $\begin{array}{l}268 \\
(4.9)\end{array}$ \\
\hline NMI2 & $\begin{array}{c}26 \\
(10.4)\end{array}$ & $\begin{array}{r}209 \\
(9.9)\end{array}$ & & $\begin{array}{r}240 \\
(4.9)\end{array}$ & $\begin{array}{r}287 \\
(6.4)\end{array}$ & $\begin{array}{r}305 \\
(5.5)\end{array}$ \\
\hline $\mathrm{IOH} 1$ & $\begin{array}{l}312 \\
(9.2)\end{array}$ & $\begin{array}{c}403 \\
(10.9)\end{array}$ & $\begin{array}{c}354 \\
(9.0)\end{array}$ & & & $\begin{array}{c}125 \\
(7.8)\end{array}$ \\
\hline IMI1 & $\begin{array}{c}491 \\
(7.3)\end{array}$ & $\begin{array}{l}458 \\
(9.4)\end{array}$ & $\begin{array}{c}562 \\
(7.5)\end{array}$ & $\begin{array}{r}199 \\
(5.9)\end{array}$ & & \\
\hline IMI3 & $\begin{array}{c}434 \\
(7.0)\end{array}$ & $\begin{array}{r}459 \\
(9.0)\end{array}$ & $\begin{array}{c}528 \\
(7.5)\end{array}$ & $\begin{array}{c}100 \\
(5.0)\end{array}$ & $\begin{array}{c}125 \\
(7.6)\end{array}$ & \\
\hline
\end{tabular}


Fig. 5 | Comparison of transcriptomes between invasive and native $\boldsymbol{P}$. australis genotypes. a, Details on genotype collections of invasive (I) and native (N) subspecies from locations marked on Fig. 1c. b, A maximum likelihood tree based on deduced protein sequences of 5,394 homologous gene groups. Protein sequences were deduced from transcriptomes de novo assembled using RNA-seq reads derived from the six $P$. australis genotypes shown in (a) as well as the reference gene models (marked with "G"). Publicly available transcriptome and genome sequences were used for Arundo donax ${ }^{69}$ and Sorghum bicolor, respectively. All branches were 100\% supported by 100 bootstrap tests. c, RNA-seq reads from leaf and rhizome tissues of the six genotypes were aligned to the $P$. australis reference genome. Principal Component Analysis separated different tissues and genotypes. d, Differentially Expressed Genes (DEGs) showing significant (adjusted $p$-value $<0.05$ and fold-difference $\geq 2$ ) changes in basal-level expression in leaf (L) and rhizome (R) were identified between all pairs of genotypes. In the diagonal plot, the circle in each cell represents the proportion of DEGs among 64,857 P. australis reference gene models in which the genotype in the column (red) shows higher basal-level expression than the clone in the row (blue). e-f, GO terms "defense response" (e) and "transmembrane transport" (f) showed enrichment among DEGs in which invasive and native genotypes showed higher basallevel expression in pairwise comparisons, respectively. Each cell shows the number and percentage of DEGs annotated with the GO term among all DEGs showing higher basal-level expression in the genotype specified by the column (red) compared to the genotype by the row (blue). Adjusted p-values of enrichment were calculated compared to a background of 41,595 reference gene models annotated with any GO term and represented as a color heatmap. 


\section{a}

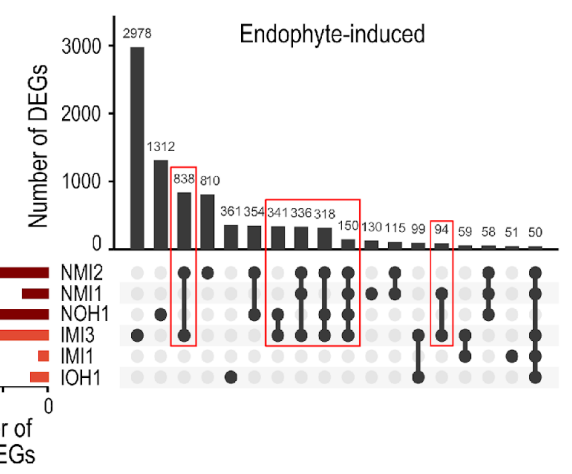

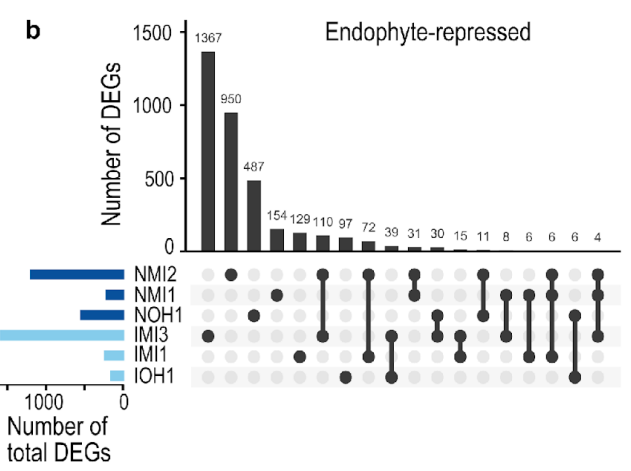

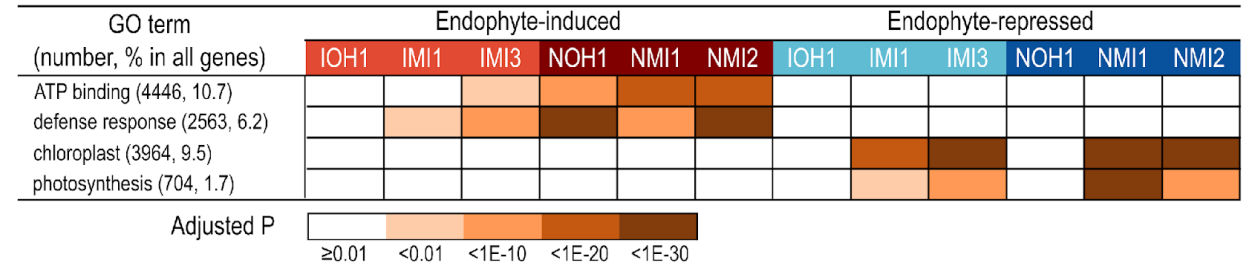

d
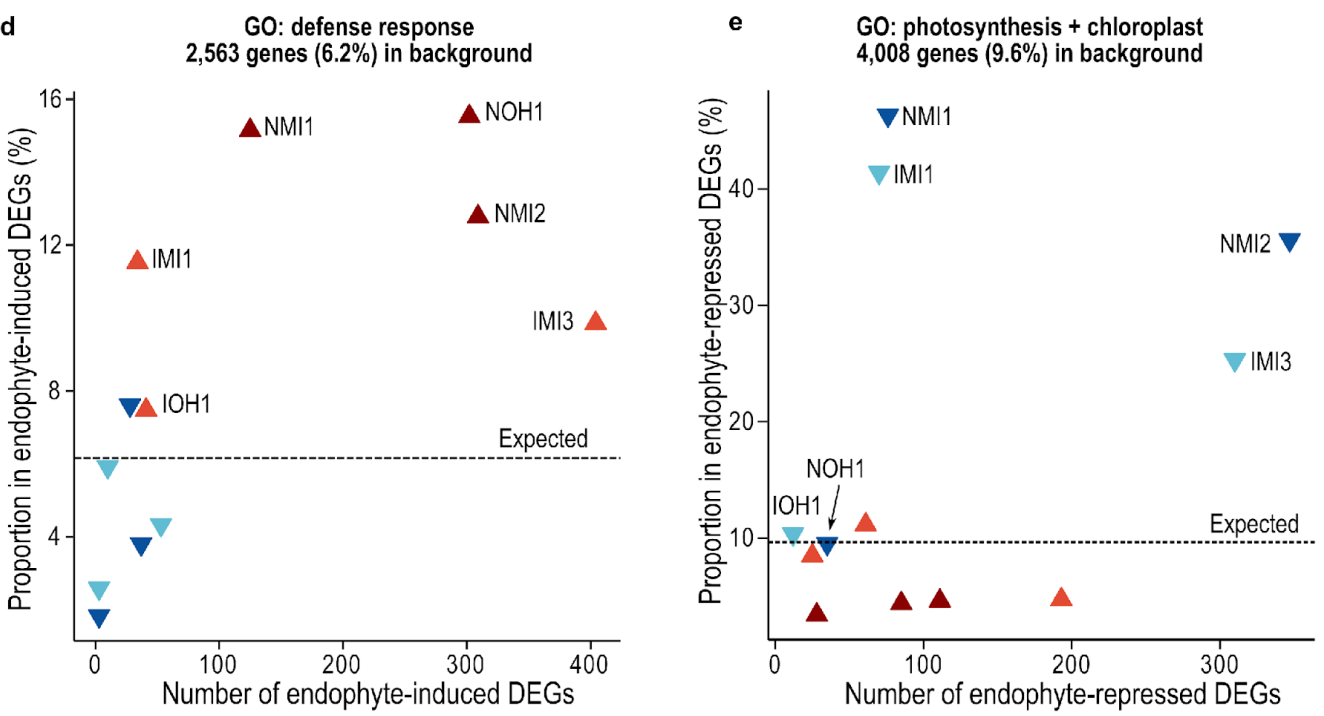

Fig. 6 | Transcriptome responses to Alternaria alternata fungal endophyte inoculation of invasive and native $\boldsymbol{P}$. australis genotypes. a-b, Upset plots showing the number of shared and unique endophyte-induced (a) and repressed (b) DEGs among the six invasive and native genotypes. Red boxes show endophyte-induced DEGs shared between IMI3 genotype and a native genotype. c, The pattern of GO enrichment in six clones involving the largest number of endophyte-induced DEGs (represented by GO terms "ATP binding" and "defense response") and endophyte-repressed DEGs (represented by GO terms "chloroplast" and "photosynthesis"). d-e, Number and percent proportion of endophyte-induced (upward triangles) or repressed (downward triangles) DEGs annotated with the GO term "defense response" (d) or either of the GO terms "photosynthesis" and "chloroplast" (e). The percent proportions in the entire $P$. australis gene models are marked with dashed lines as the expected value when there is no enrichment. 
Contents of Supplementary Materials (provided separately)

\section{Supplementary Methods}

Supplementary Table 1 | Proportion of transposable elements in the Phragmites australis reference genome

Supplementary Table 2 | Monocot genomes for comparative analyses

Supplementary Table 3 | Comparison of orthologous gene copy numbers among $P$. australis and five monocot genomes

Supplementary Table 4 | De novo assembled $P$. australis transcriptomes

Supplementary Table 5 | Alignments of RNA-seq reads derived from invasive and native genotypes to the $P$. australis reference genome

Supplementary Fig. 1 | Distribution of non-reference allele frequencies indicates a functionally diploid genome of the $P$. australis genotype used for the reference genome assembly.

Supplementary Fig. 2 | Properties of synteny blocks identified within the P. australis genome.

Supplementary Fig. 3 | The fifty largest synteny blocks detected within the $P$. australis genome.

Supplementary Fig. 4 | RNA-seq analysis comparing basal-level expression among native and invasive $P$. australis genotypes.

Supplementary Fig. 5 | An example network of Gene Ontology (GO) terms enriched among $P$. australis reference genes with higher basal-level expression in an invasive genotype.

The following datasets are available at the USGS repository (https://doi.org/10.5066/P91XSF3):

Supplementary Dataset 1. $P$. australis version 1.0 reference gene models and syntelog pairs

Supplementary Dataset 2. Ortholog groups identified among $P$. australis and five monocots

Supplementary Dataset 3. GO clusters enriched among $P$. australis ortholog groups showing conserved or increased gene copy numbers due to $P$. australis-specific duplications

Supplementary Dataset 4. RNA-Seq raw read counts and expression rank percentiles for $P$. australis gene models for invasive and native genotypes.

Supplementary Dataset 5. RNA-Seq results comparing basal-level expression of $P$. australis genes among invasive and native genotypes

Supplementary Dataset 6. GO terms enriched among $P$. australis genes with significantly different basal-level expression among invasive and native genotypes.

Supplementary Dataset 7. RNA-Seq results for endophyte-responses of $P$. australis genotypes

Supplementary Dataset 8. GO terms enriched among $P$. australis genes whose expression were significantly induced or repressed by endophyte inoculation. 